\title{
El índice de masa corporal está asociado con el adenocarcinoma de esófago y cardias
}

Lagergren J, Berstrom R, Nyrén O.Ann Intern Med.1999;130:883-890.

\section{Objetivo}

Examinar la asociación entre el índice de masa corporal (IMC) y el desarrollo de adenocarcinoma de esófago y cardias gástrico.

\section{Diseño}

Estudio caso-control poblacional.

\section{Lugar}

Suecia, de 1995 a 1997.

\section{Pacientes}

Residentes suecos nacidos en ese país de menos de 80 años de edad que desarrollaron adenocarcinoma de cardias o esófago y carcinoma epidermoide de esófago (constituyeron el $83 \%$, $87 \%$ y $73 \%$ respectivamente de los casos de la base de datos). Los 820 controles poblacionales representaron el $73 \%$ de los elegidos originalmente.

\section{Evaluación de los factores de riesgo}

Los participantes respondieron encuestas sobre su peso a los 20 años de edad y sobre el peso 20 años antes de la entrevista; además compararon su estructura corporal de 20 años atrás con nueve pictogramas distintos, que iban desde la delgadez hasta la obesidad.

También compararon la cantidad de comida usualmente ingerida utilizando fotografías; y se estimó la cantidad de ejercicio físico en cuatro niveles diferentes.

\section{Medición de resultados principales}

Los 195 hospitales involucrados en el diagnóstico y tratamiento de estos pacientes colaboraron con el registro, además se utilizaron los registros de cáncer regionales para identificar los casos perdidos.

Los controles fueron seleccionados de manera aleatorizada en estratos por edad y sexo para garantizar una distribución similar a la de los casos.

\section{Resultados principales}

Se realizaron ajustes por edad, sexo, tabaquismo, ingesta de alcohol, escolaridad, síntomas de reflujo, ingesta de verduras y frutas, cantidad de comida ingerida y actividad física. Las variables se consideraron en forma categórica y continua.

Se encontró asociación dosis dependiente entre IMC y adenocarcinoma esofágico, odds ratio $7.6(95 \%$ IC, 3.8-15.2) entre personas en el cuartilo mayor respecto del menor y de $16.2(95 \%$ IC, 6.3-41.4) para obesos (IMC > 30). El odds ratio para pacientes con adenocarcinoma de cardias fue $2.3(95 \%$ IC, 1.5-3.6) en el cuartilo superior y $4.3(95 \%$ IC, $2.1-8.7)$ entre los obesos.

No hubo asociación entre IMC y carcinoma epidermoide de esófago.

\section{Conclusiones}

Hay fuerte asociación entre adenocarcinoma de esófago e IMC que no está explicada por sesgos o confundidores, sin embargo, el efecto carcinogénico queda por ser aclarado.

\section{Comentario}

Este estudio caso-control encontró una fuerte asociación dosis dependiente entre el IMC y el adenocarcinoma de esófago, y en menor medida, aunque estadísticamente significativa, otra con el adenocarcinoma de cardias gástrico. Este hallazgo no es nuevo, ya había algunos estudios similares que sẹnalaban lo mismo mientras que otros lo ponían en duda.

Los autores refieren que esta discrepancia podría deberse a que el presente estudio tuvo la oportunidad de entrevistar a todos los pacientes luego del diagnóstico, es decir que no hubo pérdida de casos; además de haber inquirido sobre el peso corporal 20 años antes de la entrevista, con lo cual el desarrollo del cáncer no pudo haberlo alterado.

El sesgo de recuerdo es posible en este tipo de diseño, esto es que cada paciente recordará con mayor facilidad exposición a un determinado factor causal una vez conocido el diagnóstico, pero debe tenerse en cuenta el hecho que la probable asociación entre IMC y cáncer esofágico no es conocida por el público en general. Además hubo asociación "dosis" dependiente entre ambas variables, con lo cual es improbable que tanto pacientes con sobrepeso moderado como obesos hayan incurrido de igual manera en el mismo sesgo.

Otro punto a favor del estudio es que los pacientes no estaban al tanto del tipo histológico de tumor, a pesar de lo cual hubo asociación entre adenocarcinoma y no con carcinoma epidermoide de esófago.

Se realizaron ajustes sobre todos los factores predisponentes sospechados; y aún si hubiere habido reflujo asintomático, la diferencia se mantiene luego de un análisis de sensibilidad.

Finalmente, tengamos en cuenta que esta investigación fue realizada en individuos blancos nacidos en Suecia, lo cual deja el interrogante sobre cómo se comportarán otras poblaciones con respecto a este hallazgo. Otro aspecto a preguntarse es el mecanismo por el cual el aumento de la masa corporal incide en el desrrollo de cáncer de esófago, y si el verdadero mecanismo es este o u otro relacionado que aún no podemos identificar claramente. Dado el aumento de la incidencia de adenocarcinoma de esófago a límites casi epidémicos, probablemente sea necesario un estudio de cohortes que permita identificar claramente los factores predisponentes 1 . 